\title{
Locus of rotation effects in recognition
}

\author{
PATRICK CAVANAGH \\ Université de Montréal, Montréal, Québec, Canada HSC $3 J 7$
}

\begin{abstract}
Test-letter orientation has different effects on positive- and negative-recognition reaction time; furthermore, when two test letters are presented simultaneously, the effect of nontarget test orientation differs for positive and negative responses. Orientation is seen to affect the output but not the duration of the comparison stage; the continuously variable comparison output then influences the duration of the decision stage. These data show that the processing of rotated letters in recognition does not involve mental rotation.
\end{abstract}

The time required to discriminate between a normal letter and its mirror image increases linearly with rotation of the letter from the upright position (400-msec/180-deg rotation, Cooper \& Shepard, 1973). This evidence suggests that subjects mentally rotate the letter to the upright in order to make the decision and that the speed of this mental rotation is rather slow. A recent study by Corballis and Zbrodoff (Note 1) indicates that this slow process may be necessitated by the difficulty of the normal vs mirror image decision; when subjects have only to name a letter, the effect of rotation on naming latency, while remaining linear, is much smaller in magnitude (35-msec/180-deg rotation). This effect might be predicted by mechanisms such as rapid mental rotation, rotation-independent feature detection, or spatial transforms with broad orientation tuning.

Using Sternberg's (1969) additive factor method, an experiment was devised to determine the level of processing at which rotation has its effect and, consequently, which mechanism is most appropriate. The additive factor technique proposes that the duration of a response is the additive sum of the durations of the component stages of the response. If a given variable affects one and only one stage, then any other variable that interacts with it must also influence that stage. Using this approach, Sternberg has divided the recognition process into four independent, sequential stages: encoding, comparison, decision, and response execution.

Localizing the rotation effect with this method requires sufficient factors to allow the separation of the various stages. The naming latency task of Corballis and Zbrodoff (Note 1) is inadequate in this respect, as each letter receives only one response and the memory access stage involves a fixed memory set. A visual recognition task (Atkinson, Holmgren, \& Juola, 1969) was therefore designed having two levels of visual set size (one or two letters, simultaneously presented) and two response types (positive if the visual set included the

This research was supported by the National Research Council of Canada. Requests for reprints should be sent to Patrick Cavanagh, Département de Psychologie, Université de Montréal, Montréal, Québec, Canada. previously presented target letter, negative if not). Set size and response type do not interact (Atkinson et al., 1969) and are assumed to affect the comparison and decision stages, respectively.

Rotation was introduced by allowing the visual set letters to appear independently in any of four orientations $(0,90,180$, or $270 \mathrm{deg})$. If the orientation of the test letters is normalized in the encoding stage, there should be no interaction between response type and orientation. If such an interaction exists, a comparison stage may be assumed in which the duration of a match comparison is influenced by orientation in a different manner than is the duration of a nonmatch. The possibility of unequal match and nonmatch durations has been proposed by Burrows and Okada (1971) in the context of a serial comparison stage, and there is evidence of differential effects of rotation on match and nonmatch completion times (Arnoult, 1954). If rotation does affect the comparison duration, it must do so in the same manner for nonmatches whether the trial is positive or negative. An interaction of response type with nontarget test orientation would therefore indicate a decision stage locus for the effect of rotation on reaction time.

\section{METHOD}

\section{Subjects}

Eleven subjects were paid $\$ 10$ each for participation. All had normal or corrected-to-normal vision.

\section{Stimuli}

The stimuli were the uppercase letters $F, G, R$, and $J$ displayed on a 10 by 10 dot matrix on a CRT. The target letter was always upright, while the test letter or letters could appear in any of four orientations $(0,90,180$, or $270 \mathrm{deg})$. The letters subtended $1.5 \mathrm{deg}$ of visual angle horizontally and vertically and therefore remained unchanged in height or width at any of the four orientations. Both the target and the test letter or letters were bracketed by vertical lines on the left- and rightspaced $1.5 \mathrm{deg}$ of visual angle from the center of the adjacent letter. In the case of two test letters, they were arrayed horizontally, separated center to center by $1.5 \mathrm{deg}$.

A varied-set visual search paradigm was used, with set sizes of one and two test letters being equally represented in the 352 trials. The ratio of positive to negative trials was $6: 5$ for both set sizes. For Set Size 1, test letters appeared with equal 
frequency in each of the four orientations, while for Set Size 2, all 16 combinations of the orientations of the two test letters were equally represented. The test letter in positive trials appeared equally often at the left- or right-hand position.

The target and test letters for each of the 352 trials were chosen randomly, subject to the conditions just mentioned and to the following constraints: no more than four consecutive trials of the same response type; the same letter could not be the target on two consecutive trials; the same letter could not appear as a test in the same position and orientation on two consecutive trials.

\section{Procedure}

Each trial began with the presentation of the target letter in the upright position for $250 \mathrm{msec}$. Following a 400-msec interval, one or two test letters appeared, again for $250 \mathrm{msec}$. As soon as the subject responded, feedback was presented for $750 \mathrm{msec}$ (OK, ERREUR, or TROP LONG) along with the reaction time, if correct. A 750-msec delay preceded the start of the next trial. An experimental session took approximately $20 \mathrm{~min}$ and a $30-\mathrm{sec}$ rest period was given halfway through the session.

The subject sat at a table facing the CRT display with each forefinger resting on a response key. All subjects made positive responses with their dominant hand (the right hand in every case) and negative responses with their nondominant hand. The experimenter explained that the first letter presented on each trial (the target) would be followed quickly by one or two test letters that could be at various orientations, that a positive response should be given if the target letter matched a test letter, irrespective of orientation, and that a negative response should be given otherwise. The subject was asked to respond as quickly and accurately as possible.

Each subject received a practice session of 20 trials followed by two experimental sessions, both using the same sequence of 352 trials. A minimum of $15 \mathrm{~min}$ rest was given between the two experimental sessions.

\section{RESULTS}

The error rate was $4.25 \%$ (5.45 in Session 1 and 3.05 in Session 2), $4.51 \%$ for positive trials and $4.04 \%$ for negatives.

Figure 1 shows the correct positive and negative reaction times (RTs) for Set Size 1 as a function of test-letter orientation. A large rotation effect is evident for positive responses, with the shape and magnitude of the effect being very similar to that reported by Corballis and Zbrodoff (Note 1) for naming latency. The negative RTs, on the other hand, show little variation with rotation and the interaction of Response Type by Rotation is significant at the .01 level. These data thus indicate that the influence of test-letter orientation extends beyond the encoding stage.

The analysis for Set Size 2 is complicated by the confounding of left- and right-hand test orientation with target position for positive trials. To separate the confounded variables, rotation can be represented as a single factor by using the column and row means of the left orientation by right orientation data matrix. The data showing the effect of the four orientations of the lefthand test letter would then be the RTs averaged across all four orientations of the right-hand test, and vice versa.

This recombination of the data permits the orienta-

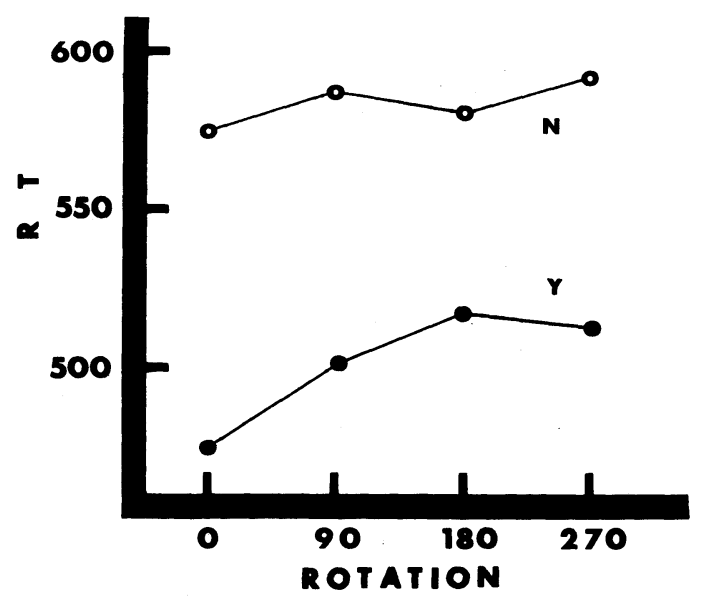

Figure 1. Mean correct positive $(\mathrm{Y})$ and negative $(\mathrm{N})$ reaction times in milliseconds for Set Size 1 as a function of test orientation.

tion factor to be analyzed as a function of the type of test letter-negative, positive target, and positive nontarget-and each of these for left- and right-hand presentation. However, the row and column means are only valid representations of the right and left letter rotation effects if the two effects are additive, that is, if there is no interaction between the two. This additivity is also required by serial models of the comparison stage where the possible effects of rotation on match and/or nonmatch comparison durations should be additive. These models would allow a right by left orientation interaction if it were located in the encoding stage, in which case it should be seen in the data when averaged over response type.

A preliminary analysis of variance was conducted to test for these interactions. In the Set Size 2 data, averaged over response type, there is no interaction of Right-Hand Test Orientation by Left-Hand Test Orientation $[F(9,90)=1.83, p<.075]$. This additivity restricts serial comparison models to a prediction of no interaction in any of the individual response conditions. The Right by Left Orientation interaction was therefore tested in the three response conditions independently for each session with an experimentwise significance level based on the Bonferroni inequality (Perlmutter $\&$ Myers, 1973). The $F$ ratios on 9 and 90 degrees of freedom for negative, positive target left, and positive target right data are, in Session 1, 1.43, 2.07, and 4.22 , respectively, and in Session 2, 2.19, 0.29, and 1.41 , respectively. Only the interaction for positive target right data in Session 1 reaches significance $(p<.001)$. None of the others exceeds an experimentwise significance level of $p<.18$.

Overall, the additivity of right- and left-hand rotation effects in the combined data and the appearance of an interaction in only one of six conditions indicate that the additivity requirement for the main analysis based on row and column means is not grossly violated. The 
importance of this one interaction for serial models of the comparison process is open to interpretation.

In the main analysis of variance, the data cells derived from the row and column means of the right test orientation by left test orientation matrix were analyzed as a function of four variables: test type $(\mathrm{T})$, having three levels, negative $(\mathrm{N})$, positive target $(\mathrm{Y})$, and positive nontarget $\left(\mathrm{Y}^{\prime}\right)$; session $(\mathrm{P})$; rotation $(\mathrm{R})$; and laterality (L). Because of the structure of the data, the test type and laterality factors are meaningful only in their interactions with rotation. The negative RTs (N) necessarily have the same means for the left- and righthand letters, while the mean left-hand positive target RT equals the mean right-hand positive nontarget RT, and vice versa. The interactions of these factors with orientation, on the other hand, are not constrained in any way, and these are the interactions of main interest here.

Figure 2 shows the RT data for Set Size 2 as a function of the rotation of negative, positive target, and positive nontarget test letters averaged over left- and right-hand presentation. The interaction of Test Type $\left(\mathrm{N}, \mathrm{Y}\right.$, and $\left.\mathrm{Y}^{\prime}\right)$ by Rotation is highly significant $[\mathrm{F}(6,60)=$ $5.33, \mathrm{p}<.001]$. Taking the negative RTs as the control condition and the two types of positive RTs as treatments (Perlmutter \& Myers, 1973), the interaction of the contrast of Negative vs Positive Target ( $\mathrm{N}$ vs $\mathrm{Y}$ ) by Rotation is also significant $[\mathrm{F}(3,60)=5.80, \mathrm{p}<.01]$, as is that of Negative vs Positive Nontarget ( $\mathrm{N}$ vs $\mathrm{Y}^{\prime}$ ) by Rotation $[F(3,60)=5.61, p<.01]$. None of the six remaining interactions among rotation and session, laterality, or test type reaches a significance level of $\mathrm{p}<.10$.

Of the interactions that were significant, that between the rotation effect for negative and positive targets ( $\mathrm{N}$ vs $\mathrm{Y}$ in Figure 2) again indicates that rotation must influence processing beyond the encoding stage.

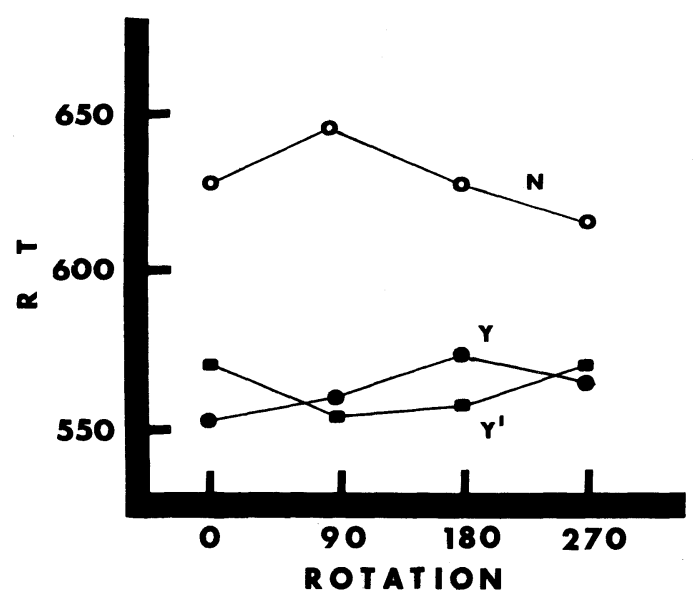

Figure 2. Set Size 2 RT as a function of rotation of negative $(N)$, positive target $(Y)$, and positive nontarget $\left(Y^{\prime}\right)$ test letters averaged over left and right presentations.
On the other hand, the interaction between the rotation effect for negative and positive nontargets ( $\mathrm{N}$ vs $\mathrm{Y}^{\prime}$ in Figure 2) indicates that the influence of rotation also extends beyond the comparison stage.

\section{DISCUSSION}

A rotation effect for positive nontargets which is practically the inverse of that for negative nontargets cannot have its locus in either the encoding or the comparison stage. If rotation necessarily affects the decision stage, the concept of a binary output (match or no match) from the comparison process must be rejected, as such a variable can carry no information concerning rotation. If the variable is therefore continuous, an appropriate decision stage can be constructed (Bregman, Note 2), but it is quite different from the binary decision stage described by Sternberg (1969).

First, the continuous comparison stage output variable must be translated in the decision stage to a binary output, requiring, therefore, a decision criterion. Second, since the effect of nontarget orientation on RT cannot be located in the comparison stage, the nontarget comparison must be able to contribute to the comparison output variable and the value of that variable must be able to influence the decision stage duration. It is reasonable to propose that decision time is some decreasing function of the distance of the comparison variable from the decision criterion. The decision stage duration is therefore influenced by the number of nontargets, each able to contribute to the comparison variable. The comparison stage is thus not the sole stage affected by the size of the test set. Consequently, factors that interact with set size are not necessarily acting on the comparison stage.

This decision stage makes a specific prediction concerning the relationship between the effect of nontarget rotation on positive and negative RT. Consider that the decision-time component of RT decreases monotonically as the distance between the comparison variable and the decision criterion increases. The contribution of a nontarget test to the comparison variable must be independent of the ensuing response; consequently, a nontarget orientation that increases the comparison variable will increase the separation from the criterion when the variable lies above the criterion, but decrease the separation when it lies below. Nontarget orientation must therefore have opposite effects on positive (comparison variable above the criterion) and negative (comparison variable below) RTs. The inverse relation between negative and positive nontarget rotation effects seen in Figure 2 ( $N$ vs $\mathrm{Y}^{\prime}$ ) now becomes meaningful: A nontarget orientation that slows a negative response should speed a positive one.

Since the locus of the RT effect of nontarget orientation is the decision stage, the decision stage is necessarily a source of RT variation influenced by set size. Recent reports of the interaction of response factors with set size in memory search tasks (Egeth, Marcus, \& Bevan, 1972; Howard, 1975) indicate that the equivalent situation may also exist in that paradigm. Shinar and Owen (1973) have, in fact, reported a significant three-way interaction involving rotation, set size, and response type in a memory search task. Although they concluded that their data supported a comparison stage locus for the RT effect of rotation, it is obvious that the involvement of response type in the three-way interaction specifically rules out the comparison stage and again indicates a decision stage locus.

Finally, although the data considered here place the RT locus of rotation in the decision stage, the origin of the orientation effect lies in the comparison stage-orientation affects the value of the comparison stage output but not the duration of the stage. Any mechanism proposing to explain the effect of the orientation of test letters as an increase in comparison duration (as would a mental rotation operation) can, therefore, 
be ruled out. A comparison process is called for whose output, but not duration, is a function of orientation. Filter mechanisms based in transform or feature domains with very broad orientation tuning are suggested.

\section{REFERENCE NOTES}

1. Corballis, M. C., \& Zbrodoff, J. Latency of identifying rotated letters and digits. Unpublished manuscript, 1975. (Available from author, Department of Psychology, McGill University, Montréal, Québec, Canada.)

2. Bregman, A. Personal communication, December 1975.

\section{REFERENCES}

Arvoult, M. D. Shape discrimination as a function of the angular orientation of the stimuli. Journal of Experimental Psychology, 1954, 47, 323-328.

Atrinson, R. C., Holmgren, J. E., \& Juola, J. F. Processing time as influenced by the number of elements in a visual display. Perception \& Psychophysics, 1969, 6, 321-326.

BurRows, D., \& OKADA, R. Serial position effects in high- speed memory search. Perception \& Psychophysics, 1971, 10, 305-308.

Cooper, L. A., \& Shepard, R. N. The time required to prepare for a rotated stimulus. Memory \& Cognition, 1973, 1, 246-250.

Egeth, H., Marcus, N., \& Bevan, W. Target-set and response-set interaction: Implications for models for human information processing. Science, 1972, 176, 1447-1448.

HowARD, J. H. The attentional demands of negation in a memory-scanning task. Memory \& Cognition, 1975, 3, 319-324.

Perlmutter, J., \& Myers, J. L. A comparison of two procedures for testing multiple contrasts. Psychological Bulletin, 1973, 79, 181-184.

ShINAR, D., \& OWEN, D. H. Effects of form rotation on the speed of classification: The development of shape constancy. Perception \& Psychophysics, 1973, 14, 149-154.

STERnBerg, $S$. The discovery of processing stages: Extensions of Donders' method. In W. G. Koster (Ed.), Attention and performance II. Acta Psychologica, 1969, 30, 276-315.

(Received for publication March 21, 1977.) 